\title{
Fat depot-related differences in gene expression, adiponectin secretion, and insulin action and signalling in human adipocytes differentiated in vitro from precursor stromal cells
}

\author{
S. Perrini • L. Laviola • A. Cignarelli • M. Melchiorre • \\ F. De Stefano • C. Caccioppoli • A. Natalicchio • \\ M. R. Orlando • G. Garruti • M. De Fazio • \\ G. Catalano • V. Memeo • R. Giorgino • F. Giorgino
}

Received: 3 August 2007 / Accepted: 3 September 2007 / Published online: 25 October 2007

(C) Springer-Verlag 2007

\begin{abstract}
Aim/hypothesis The distinct metabolic properties of visceral and subcutaneous adipocytes may be due to inherent characteristics of the cells that are resident in each fat depot. To test this hypothesis, human adipocytes were differentiated in vitro from precursor stromal cells obtained from visceral and subcutaneous fat depots and analysed for genetic, biochemical and metabolic endpoints.

Methods Stromal cells were isolated from adipose tissue depots of nondiabetic individuals. mRNA levels of adipocytespecific proteins were determined by real-time RT-PCR.
\end{abstract}

Electronic supplementary material The online versin of this article (doi:10.1007/s00125-007-0841-7) contains supplementary material, which is available to authorised users.

S. Perrini $\cdot$ L. Laviola $\cdot$ A. Cignarelli $\cdot$ M. Melchiorre

F. De Stefano $\cdot$ C. Caccioppoli $\cdot$ A. Natalicchio $\cdot$ M. R. Orlando

G. Garruti $\cdot$ R. Giorgino $\cdot$ F. Giorgino

Department of Emergency and Organ Transplantation, Section of Internal Medicine, Endocrinology and Metabolic Diseases, University of Bari School of Medicine,

Bari, Italy

M. De Fazio • G. Catalano • V. Memeo

Department of Emergency and Organ Transplantation, Section of General Surgery, University of Bari School of Medicine,

Bari, Italy

F. Giorgino $(\bowtie)$

Department of Emergency and Organ Transplantation, Section of Internal Medicine, Endocrinology and Metabolic Diseases,

University of Bari,

Piazza Giulio Cesare, 11,

I-70124 Bari, Italy

e-mail: f.giorgino@endo.uniba.it
Insulin signalling was evaluated by immunoblotting with specific antibodies. Glucose transport was measured by a 2-deoxy-glucose uptake assay. Adiponectin secretion in the adipocyte-conditioned medium was determined by a specific RIA.

Results With cell differentiation, mRNA levels of PPARG, $C / E B P \alpha$ (also known as $C E B P A$ ), $A P 2$ (also known as GTF3A), GLUT4 (also known as SLC2A4) were markedly upregulated, whereas GLUT1 (also known as $S L C 2 A 1$ ) mRNA did not change. However, expression of $C / E B P \alpha$, $A P 2$ and adiponectin was higher in subcutaneous than in visceral adipocytes. By contrast, adiponectin was secreted at threefold higher rates by visceral than by subcutaneous adipocytes while visceral adipocytes also showed two- to threefold higher insulin-stimulated glucose uptake. Insulininduced phosphorylation of the insulin receptor, IRS proteins, Akt and extracellular signal-regulated kinase-1/2 was more rapid and tended to decrease at earlier time-points in visceral than in subcutaneous adipocytes.

Conclusions/interpretation Subcutaneous and visceral adipocytes, also when differentiated in vitro from precursor stromal cells, retain differences in gene expression, adiponectin secretion, and insulin action and signalling. Thus, the precursor cells that reside in the visceral and subcutaneous fat depots may already possess inherent and specific metabolic characteristics that will be expressed upon completion of the differentiation programme.

Keywords Adiponectin · Akt · Erk .

Extracellular signal-regulated kinase - Glucose uptake .

Insulin signalling · Subcutaneous fat $\cdot$ Visceral fat 


$\begin{array}{ll}\begin{array}{ll}\text { Abbreviations } \\ \text { C/EBP } \alpha\end{array} & \begin{array}{l}\text { CCAAT/enhancer binding protein (C/EBP), } \\ \text { alpha }\end{array} \\ \text { Erk } & \begin{array}{l}\text { extracellular signal-regulated kinase } \\ \text { insulin receptor } \\ \text { IR }\end{array} \\ \text { PTEN } & \begin{array}{l}\text { in multiple advanced cancers 1) } \\ \text { protein tyrosine phosphatase 1B }\end{array} \\ \text { PTP-1B } & \text { SH2 containing inositol phosphatase } \\ \text { SHIP-2 } & \end{array}$

\section{Introduction}

By regulating triacylglycerol metabolism and secreting a variety of cytokines and hormones with pleiotropic effects on multiple tissues, adipose tissue has an enormous capacity to regulate fuel utilisation, energy homeostasis and cardiovascular function. Dysfunctional adipose tissue, clinically evident as excessive fat mass (obesity) or abnormally reduced fat (lipodystrophy), leads to impaired glucose and lipid metabolism, insulin resistance and type 2 diabetes, as well as to increased risk of cardiovascular disease. Indeed, the distribution of body fat appears to be more important than the total amount of fat. Abdominal, and in particular visceral, adiposity shows the closest association with metabolic and cardiovascular diseases [1, 2]. An excess of visceral fat may promote decreased insulin effects on glucose uptake, NEFA re-esterification and inhibition of lipolysis [3]. Conversely, surgical elimination of visceral fat resulted in dramatic improvements in insulin sensitivity in rodents [4] and humans [5], while removal of subcutaneous fat did not prevent insulin resistance and metabolic abnormalities $[6,7]$.

The adverse metabolic impact of visceral fat has been attributed to distinct biological properties of adipocytes in this particular fat depot compared with those in other sites, including differences in metabolic responses, gene expression, adipokine secretion and insulin action [8,9]. For example, the lipolytic effect of catecholamines was shown to be more pronounced and the anti-lipolytic effect of insulin weaker in omental than in subcutaneous adipocytes [10]. Differences in the secretion of cytokines, such as adiponectin, plasminogen activator inhibitor, type I (PAI-1), IL-6 and leptin, between the two abdominal fat compartments have also been extensively described [9, 11]. Recently, we investigated insulin signalling in different fat depots in humans in vivo and demonstrated higher, more rapid and transient activation of the insulin signalling cascade in visceral than in subcutaneous fat [12]. These observations are in line with the reportedly greater effect of insulin in stimulation of glucose uptake in visceral fat, both in humans in vivo [13] and in isolated human adipocytes in vitro [14].
It should be recognised, however, that information on the metabolic properties of adipose tissue largely derives from studies in whole fat tissue in vivo, adipose tissue fragments ex vivo or isolated mature adipocytes in vitro, thus probably reflecting the influence of environmental factors in the tissue of origin. These experimental conditions cannot clarify whether the depot-related specificity of adipose cells is due to their innate characteristics or, alternatively, to extrinsic factors, such as tissue microenvironment, local circulation, local innervation and/or heterogeneity in cellularity. In this study, we used adipocytes differentiated in vitro from visceral and subcutaneous precursor stromal cells to investigate whether these cells retain the depot-related differences in gene expression, adiponectin secretion, and insulin action and signalling that are seen in intact adipose tissue and freshly isolated adipocytes.

\section{Methods}

Individuals and adipose tissue biopsies Paired abdominal subcutaneous and omental fat biopsies were obtained from 13 non-obese individuals with normal glucose tolerance (seven men, six women; aged $45 \pm 1$ years; weight $68.4 \pm$ $3.4 \mathrm{~kg}$; height, $167 \pm 3 \mathrm{~cm}$ ), who underwent elective open abdominal surgery for nonmalignant diseases (i.e. cholecystectomy, inguinal hernia, hysterectomy). They were all otherwise healthy and not taking any regular medication. All participants gave their informed consent before the surgical procedure. The study protocol was approved by the Independent Ethical Committee at the Azienda OspedalieroUniversitaria Policlinico Consorziale, University of Bari School of Medicine. Anthropometric and metabolic characterisation, including both height and weight measurements and fasting blood chemistry, were performed 2 to 3 days before the surgical procedure; see Electronic supplementary material (ESM) for additional details.

Isolation and differentiation of adipocyte precursor cells Human preadipocytes were isolated according to the method of Rodbell [15], with minor modifications, and differentiated for about 30 days in DMEM/F-12 medium supplemented with insulin, dexamethasone, triiodothyronine and rosiglitazone, in line with recently published methods for differentiation of human preadipocytes [16]; for further details on preadipocyte isolation and differentiation, see ESM. Differentiation of cells into adipocytes was assessed through morphological analysis and Oil-Red-O staining. Cells in three wells were fixed with $3.7 \%$ (vol./vol.) formaldehyde in PBS, and their triacylglycerol content was stained with $0.3 \%$ (wt/vol.) Oil-Red-O in 60\% (vol./vol.) isopropanol. After repeatedly washing with water, the differentiated adipocytes were estimated by direct counting under light microscope. 
Cells were defined as differentiated when their cytoplasm was completely filled with multiple fat droplets. All experimental analyses on adipocytes were carried out following culture for 2 days in washout DMEM/F-12 medium without insulin, dexamethasone, triiodothyronine and rosiglitazone.

RNA extraction and real-time PCR Total RNA was isolated from preadipocytes, mature adipocytes and whole adipose tissue using kits (RNeasy Mini and RNeasy Lipid Tissue Mini, respectively; Qiagen, Hilden, Germany). The mRNA levels of multiple proteins were analysed by quantitative real-time RT-PCR using a 7500 real-time PCR system (Applied Biosystems, Foster City, CA, USA), as previously described [17] (see ESM for primer sequences). The mRNA level of each target gene was normalised using $18 \mathrm{~S}$ as internal control.

Measurement of adiponectin secretion and glucose uptake Adipocytes were starved for $12 \mathrm{~h}$ in serum-free DMEM/ F10 medium containing $0.2 \%$ (wt $/ \mathrm{vol}$.) BSA and then incubated for $2 \mathrm{~h}$ in fresh medium. A fraction of the culture medium was removed for subsequent adiponectin evaluation by RIA using a specific assay kit (Linco Research, St Charles, MO, USA). The kit intra-assay and inter-assay CVs were 6.21 and $6.90 \%$, respectively. Adiponectin secretion is reported as $\mathrm{ng} / \mathrm{ml}$ of culture medium per $\mu \mathrm{g}$ of cell protein. Glucose transport measurements in human adipocytes were performed as previously described [18] (see ESM).

Analysis of insulin signalling Subcutaneous and omental adipocytes were incubated for $3 \mathrm{~h}$ in serum-free medium containing $0.5 \%$ (wt $/ \mathrm{vol}$.) BSA and then stimulated with $10 \mathrm{nmol} / \mathrm{l}$ insulin for the indicated times or left untreated. Then the cells were rapidly processed and analysed by immunoblotting, as described previously $[12,18]$ (see ESM).

Statistical analyses Differences between the two groups were analysed by the Student's $t$ test for independent samples. For the evaluation of changes in the phosphorylation profiles of the proteins investigated, one-way ANOVA and Tukey's post hoc test were used. All data are expressed as mean \pm SE. $p$ values $<0.05$ were considered to represent statistical significance.

\section{Results}

Adipocyte differentiation Adipocyte differentiation was evaluated by both morphological analysis under light microscopy and Oil-Red-O staining. No lipid droplets were
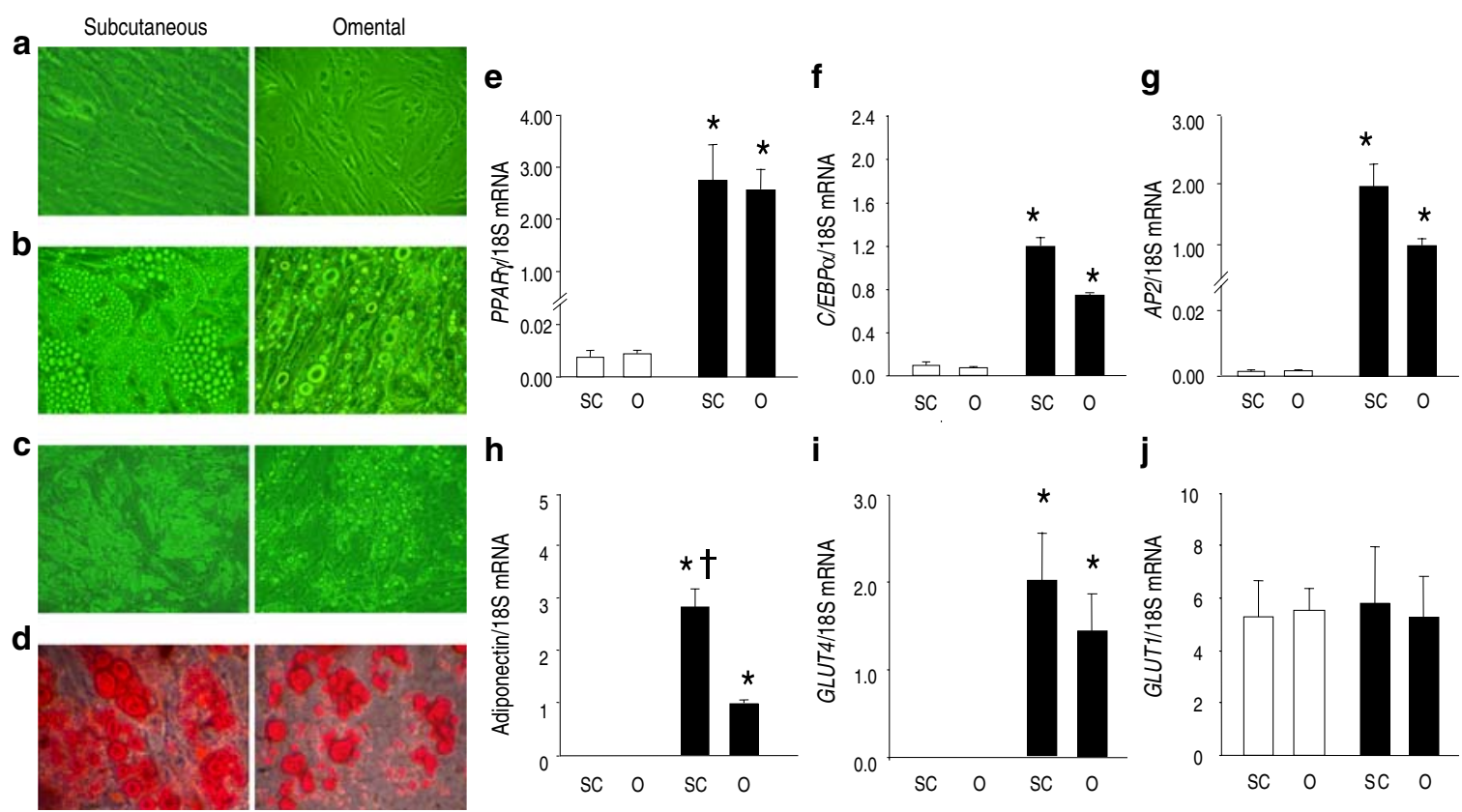

Fig. 1 Morphological and biochemical characteristics and gene expression patterns of human subcutaneous (SC) and omental (O) preadipocytes and adipocytes. a-d Primary human subcutaneous and omental preadipocytes were grown to confluence and then treated with differentiation-inducing medium as described in the Materials and methods section. Cells from one representative participant are shown. a Subcutaneous and omental preadipocytes, magnification, $\times 40$; b, c cells treated with differentiation-inducing medium for 30 days, magnification, $\times 40$ and $\times 10$, respectively; $\mathbf{d}$ adipocytes stained with

Oil-Red-O, magnification, $\times 40$. $\mathbf{e}-\mathbf{j}$ Expression of fat-specific genes in preadipocytes (white bars) and mature adipocytes (black bars). Total RNA was extracted from preadipocytes and adipocytes, and mRNA expression levels of $P P A R \gamma(\mathbf{e}), C / E B P \alpha(\mathbf{f}), A P 2(\mathbf{g})$, adiponectin (h), GLUT4 (i) and GLUT1 (j) were determined by quantitative real-time RT-PCR. The mRNA level was normalised for each target gene against $18 \mathrm{~S}$ ribosomal RNA as internal control. Values are means $\pm \mathrm{SE}$ of cells from five independent participants performed in triplicate. ${ }^{*} p<0.05$ vs preadipocytes; ${ }^{\dagger} p<0.05$ vs omental adipocytes 
present in untreated preadipocytes (Fig. 1a). However, following exposure to the differentiation medium, cells gradually accumulated large multilocular lipid droplets (Fig. 1b-d). Lipid accumulation was clearly evident in subcutaneous and omental adipocytes, and did not differ apparently between the two cell cultures (Fig. 1b-d).

$m R N A$ levels of adipocyte transcription factors, adiponectin and glucose transporters The mRNA expression levels of a
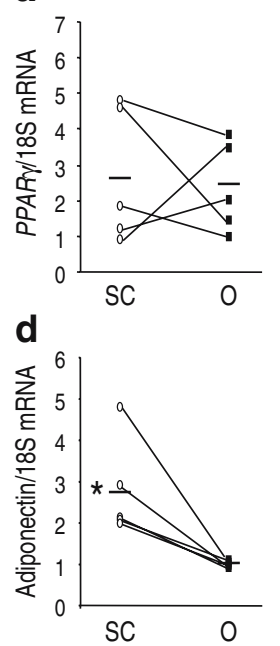

b
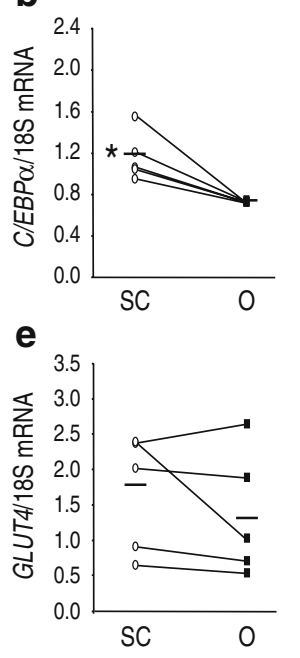

g

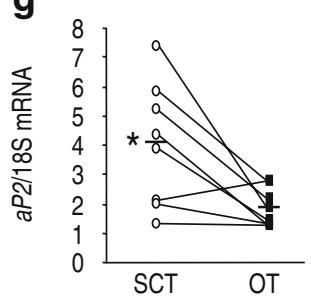

i

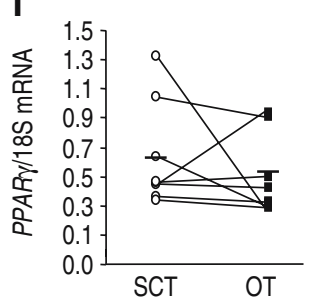

C

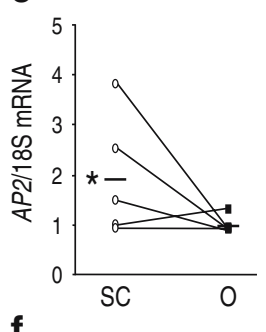

f

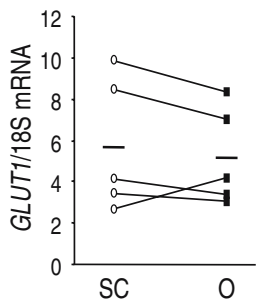

$P P A R \gamma$ (also known as PPARG), $C / E B P \alpha$ (also known as CEBPA), AP2 (also known as GTF3A), GLUT4 (also known as $S L C 2 A 4$ ) and adiponectin were undetectable in subcutaneous and omental precursor stromal cells, but were found to be increased by several fold following differentiation (Fig. 1e-i). In contrast, GLUT1 (also known as $S L C 2 A 1)$ showed similar mRNA expression levels in undifferentiated and differentiated cells (Fig. 1j). When the expression levels of these genes were examined in the differentiated adipocytes according to their fat depot-specific origin, $A P 2, C / E B P \alpha$ and adiponectin mRNAs were found to be 2.0-, 1.5- and 2.8-fold more abundant, respectively, in subcutaneous than in omental adipocytes $(p=0.021, p=$ 0.029 and $p=0.036$ vs omental cells for $A P 2, C / E B P \alpha$ and adiponectin, respectively) (Figs $1 \mathrm{e}-\mathrm{j}$ and $2 \mathrm{a}-\mathrm{f}$ ). By contrast, PPAR $\gamma$, GLUT4 and GLUT1 mRNA expression was found at comparable levels in subcutaneous and omental adipocytes ( $p=0.417, p=0.164$ and $p=0.194$ for PPAR $\gamma$, GLUT4 and GLUT1, respectively) (Figs $1 \mathrm{e}-\mathrm{j}$ and $2 \mathrm{a}-\mathrm{f}$ ). Gene expression of fat-specific genes was also analysed in freshly isolated subcutaneous and omental adipose tissue. The mRNA expression levels of $A P 2$ and adiponectin were significantly higher in subcutaneous than in omental adipose tissue ( $p=0.003$ and $p=0.005$ vs omental tissue, respectively) (Fig. 2g,h), while those of PPAR $\gamma$ and GLUT4 were found to be similarly expressed in the two fat depots $(p=0.980$ and $p=0.993$ vs omental tissue, respectively) (Fig. 2i,j).

Adiponectin secretion To determine whether depot-specific differences in adiponectin secretion could also be demonstrated in fat cells differentiated in vitro, adiponectin concentrations were measured in the culture medium of paired subcutaneous and omental cells from each participant after completion of the differentiation process. Basal adiponectin release over $2 \mathrm{~h}$ was approximately threefold higher in omental than in subcutaneous adipocytes $(p=0.001)$ (Fig. 3a).

Glucose uptake To evaluate the effects of insulin on glucose transport, subcutaneous and omental mature adipocytes were incubated with $10 \mathrm{nmol} / \mathrm{l}$ insulin for various times, and glucose transport was measured by determining the rates of 2-deoxy-D- $\left[{ }^{3} \mathrm{H}\right]$ glucose uptake. Basal glucose uptake was not different in subcutaneous and omental adipocytes $(p=0.850)$ (Fig. 3b). In subcutaneous adipocytes, a statistically significant increase in insulin-stimulated glucose uptake was evident after $30 \mathrm{~min}(p=0.017$ vs basal), with a maximum effect after 60 min (Fig. 3b,c). In omental adipocytes, a significant increase in insulininduced glucose transport was detected at all time points evaluated ( $p=0.0001$ vs basal), with a maximum effect after $60 \mathrm{~min}$ of hormone stimulation. In addition, at the 15, 30 and 60 min time-points, the magnitude of glucose transport stimulation by insulin was significantly and markedly 


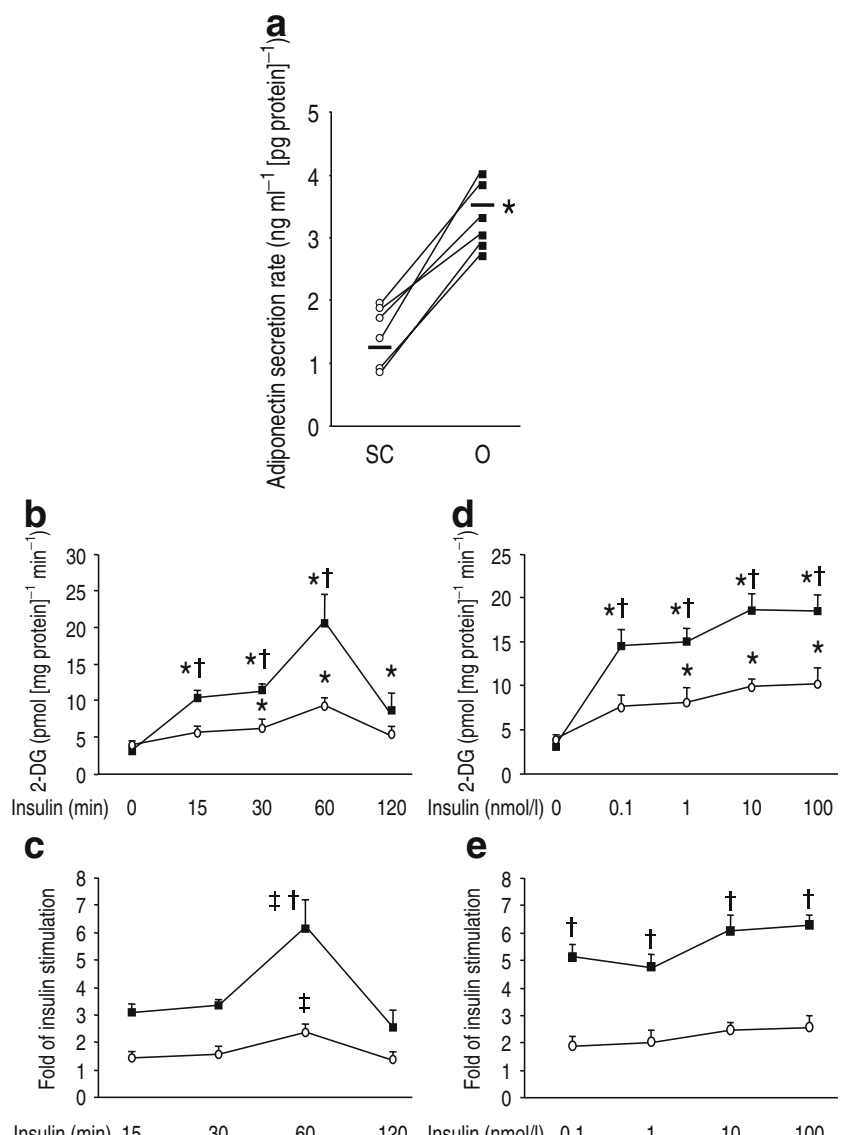

Insulin (min) $15 \quad 30 \quad 60 \quad 120$ Insulin (nmol/h) 0.1 1 10 (a) stimulated 2-deoxy-D- $\left[{ }^{3} \mathrm{H}\right]$ glucose $(2-\mathrm{DG})$ uptake $(\mathbf{b}, \mathbf{d})$ in human subcutaneous ( $\mathrm{SC}$, circles) and omental (O, squares) adipocytes differentiated in vitro from precursor stromal cells. a Adiponectin concentrations were measured in duplicate $2 \mathrm{~h}$ after incubation in serum-free DMEM/F10 medium containing $0.2 \%$ (wt/vol.) BSA, as described in the Materials and methods section. The data are plotted with lines connecting the levels of adiponectin released from paired subcutaneous and omental adipocytes of independent participants $(n=$ 6). Mean values for the overall group of subcutaneous or omental adipocytes are also indicated (horizontal bars). ${ }^{*} p<0.05$ vs SC adipocytes. b Time-course of glucose uptake rates in the absence or presence of $10 \mathrm{nmol} / 1$ insulin. Experiments from six independent participants were carried out in triplicate. Data are expressed as mean \pm SE. ${ }^{*} p<0.05$ vs basal; ${ }^{\dagger} p<0.05$ vs SC. c Fold-stimulation of glucose transport by insulin for the time-course studies. ${ }^{\dagger} p<0.05$ vs SC; ${ }^{\ddagger} p<$ 0.05 vs all other time-points. $\mathbf{d}$ Glucose uptake rates in the absence or presence of various concentrations of insulin for $60 \mathrm{~min}$. Experiments from six independent participants were carried out in triplicate. Data are expressed as means \pm SE. ${ }^{*} p<0.05$ vs basal; ${ }^{\dagger} p<0.05$ vs SC. e Fold-stimulation of glucose transport by insulin for the dose-response studies. ${ }^{\dagger} p<0.05$ vs SC

greater in omental than in subcutaneous adipocytes (sixfold vs twofold, respectively, $p<0.05$ ) (Fig. 3b,c). Next, glucose transport was determined in subcutaneous and omental adipocytes following incubation with various concentrations of insulin for $60 \mathrm{~min}$. In both cell types, insulin elicited an increase in glucose transport rates with statistically significant effects at 0.1 to $100 \mathrm{nmol} / 1$ insulin $(p=0.02$ and $p=0.001$ vs basal, for subcutaneous and omental cells, respectively) (Fig. 3d,e). All insulin doses were found to raise glucose transport to greater levels in omental than in subcutaneous adipocytes (five- to sixfold vs approximately twofold, respectively, $p<0.05$ ) (Fig. 3e). Furthermore, omental adipocytes showed a greater sensitivity to insulin stimulation, since an initial significant effect was evident at $0.1 \mathrm{nmol} / 1$ insulin, whereas in subcutaneous adipose cells glucose transport increased significantly only with $1 \mathrm{nmol} / \mathrm{l}$ insulin (Fig. 3d,e).

Insulin receptor and IRS proteins Under basal conditions, tyrosine phosphorylation of the insulin receptor (IR) $\beta$ subunit was not significantly different in subcutaneous and omental adipocytes $(p=0.96)$ (Fig. 4a). Stimulation with insulin resulted in a time-dependent increase in IR tyrosine phosphorylation in both fat cell types (Fig. 4a,b). In subcutaneous adipocytes, insulin-induced IR tyrosine phosphorylation was increased 4.2 -fold at $5 \mathrm{~min}(p=0.011$ vs basal) and reached a maximum at $15 \mathrm{~min}$ (5.5-fold), then gradually declining towards basal levels at $120 \mathrm{~min}$ (Fig. 4a,b). In contrast, insulin-stimulated IR tyrosine phosphorylation showed a rapid 5.8 -fold peak at $5 \mathrm{~min}$ ( $p=0.001 \mathrm{vs}$ basal), followed by a more rapid decline in omental adipocytes (Fig. 4a,b). The total amount of IR protein, determined by immunoblotting with anti-IR antibodies, was similar in subcutaneous and omental fat cells ( $p=0.805)$ (Fig. 4a; and data not shown).

IRS tyrosine phosphorylation, collectively evaluated by direct anti-phosphotyrosine immunoblotting, was not statistically different in subcutaneous and omental adipocytes in the basal state $(p=0.95)$ (Fig. 4c). Insulin markedly stimulated IRS tyrosine phosphorylation in the subcutaneous adipocytes, in which a peak was observed at $15 \mathrm{~min}$; then, IRS tyrosine phosphorylation showed a gradual decrease, even though it remained above basal levels for up to $120 \mathrm{~min}$ ( $p=0.017$ vs basal) (Fig. $4 \mathrm{c}, \mathrm{d}$ ). In omental adipocytes, by contrast, insulin-induced IRS tyrosine phosphorylation exhibited a maximum increase after 5 min ( $p=0.008$ vs basal), returning to basal levels after 120 min (Fig. 4c,d). Therefore, IRS tyrosine phosphorylation was significantly lower in omental than in subcutaneous adipocytes after $120 \mathrm{~min}$ of insulin stimulation ( $p=0.017$ vs subcutaneous cells) (Fig. 4d). IRS-1 protein levels were not significantly different in the two experimental cell cultures $(p=0.45)$ (Fig. 4c; and data not shown). IRS-2 protein levels could not be measured, because they were below the detection threshold of the immunoblotting analysis in human adipocytes (data not shown).

To assess whether the depot-specific phosphorylation kinetics of the IR and IRS proteins could be associated with different cellular levels of tyrosine phosphatases, the total protein content of protein tyrosine phosphatase 1B (PTP- 
a

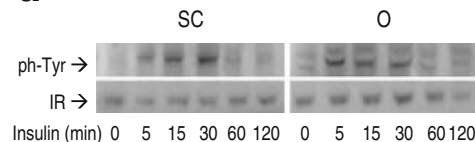

b

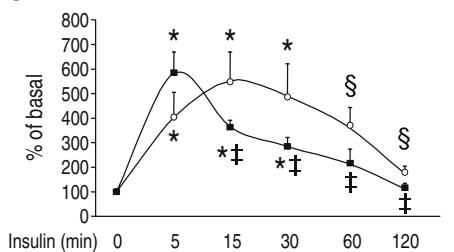

Fig. 4 Insulin receptor and IRS tyrosine phosphorylation and protein content in human subcutaneous ( $\mathrm{SC}$, circles) and omental (O, squares) adipocytes differentiated in vitro from precursor stromal cells. Adipocytes were studied in the basal state and after insulin stimulation for the indicates times. Cell lysates were subjected to immunoblotting with specific antibodies, as described in the Materials and methods section. (a) Representative immunoblots of tyrosine phosphorylation and protein content of IR. b Quantitation of IR tyrosine phosphorylation in multiple experiments. ${ }^{*} p<0.05$ vs basal; ${ }^{8} p<0.05$ vs 15 min

1B), SH2 containing inositol phosphatase (SHIP-2) and phosphatase and tensin homologue (mutated in multiple advanced cancers 1) (PTEN) was determined. However, PTP-1B, SHIP-2 and PTEN protein levels were found to be similar in subcutaneous and omental adipocytes $(p=0.67$, $p=0.72$ and $p=0.81$, respectively) (ESM Fig. 1).

a

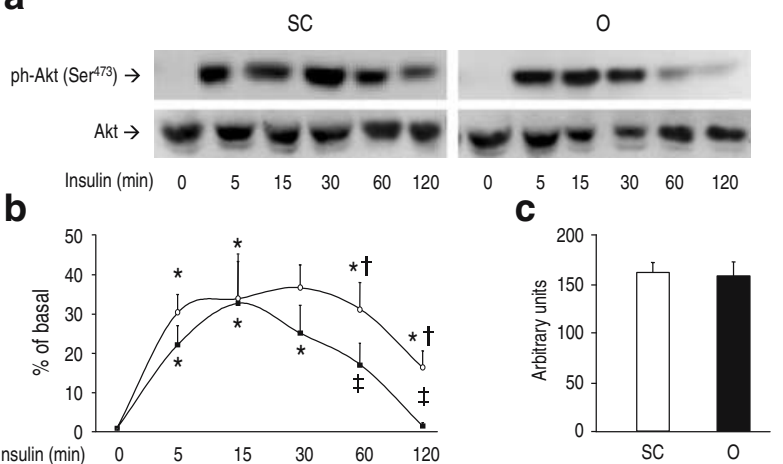

Fig. 5 Insulin stimulation of Akt phosphorylation in subcutaneous and omental adipocytes differentiated in vitro from precursor stromal cells. Subcutaneous (SC, white bars/circles) and omental (O, black bars/squares) adipocytes were studied in the absence or presence of $10 \mathrm{nmol} / \mathrm{l}$ insulin for the indicated times. Akt phosphorylation on Ser ${ }^{473}$ and protein content, respectively, were evaluated by immunoblotting with specific antibodies, as described in the Materials and methods section. Representative immunoblots (a) and the quantitation of Akt phosphorylation (b) and Akt protein content (c) are shown. For determination of total Akt protein levels, values of basal and insulinstimulated samples were pooled together. Data are expressed as mean \pm $\mathrm{SE}$ of paired subcutaneous and omental adipocytes from nine independent participants, analysed in duplicate. ${ }^{*} p<0.05$ vs respective basal; $p<0.05$ vs corresponding time-point in omental adipocytes; $p<0.05$ vs 15 min time-point in omental adipocytes

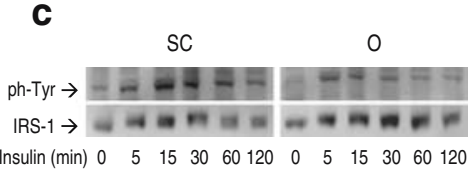

d

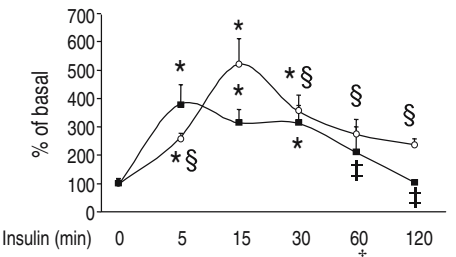

time-point in SC adipocytes; ${ }^{*} p<0.05$ vs 5 min time-point in omental adipocytes. (c) Representative immunoblots of tyrosine phosphorylation of IRS proteins and protein content of IRS-1. d Quantitation of IRS phosphorylation in multiple experiments. ${ }^{*} p<0.05$ vs basal; ${ }^{\dagger} p<$ 0.05 vs corresponding time-point in omental adipocytes; ${ }^{\S} p<0.05$ vs 15 min time-point in SC adipocytes; $p<0.05$ vs 5 min time-point in omental adipocytes. Data $(\mathbf{b}, \mathbf{d})$ are expressed as mean $\pm \mathrm{SE}$ of paired subcutaneous and omental adipocytes from nine independent participants, analysed in duplicate

Akt and extracellular signal-regulated kinase Basal Akt phosphorylation on $\mathrm{Ser}^{473}$ was not significantly different in subcutaneous and omental cells $(p=0.95)$ (Fig. 5). Insulin increased Akt phosphorylation on $\operatorname{Ser}^{473}$ by several fold in subcutaneous and omental adipocytes $(p=0.001$ and $p=0.009$ vs basal in subcutaneous and omental cells, respectively) (Fig. 5a,b). However, in subcutaneous adipocytes, phosphorylation of Akt remained significantly higher than basal both after 60 and $120 \mathrm{~min}$ of insulin stimulation (Fig. 5a,b). In contrast, in omental adipocytes Akt phosphorylation peaked at $15 \mathrm{~min}$, returning to basal levels by $120 \mathrm{~min}$ (Fig. 5b). Therefore, insulin-stimulated Akt phosphorylation was higher in subcutaneous than in omental adipocytes both at 60 and $120 \min (p=0.032$ and $p=0.036$ vs omental cells at the named time-points, respectively) (Fig. 5b). The levels of Akt phosphorylation on $\mathrm{Thr}^{308}$ were below the detection threshold of the analysis in subcutaneous and omental adipocytes. Akt protein levels were similar in subcutaneous and omental adipocytes $(p=0.92)$ (Fig. 5a,c).

Under basal conditions, phosphorylation of extracellular signal-regulated kinase (Erk)-1/2 on $\mathrm{Thr}^{202}$ and $\mathrm{Tyr}^{204}$ was not different in the subcutaneous and omental fat cell cultures $(p=0.97)$ (Fig. 6). In adipocytes derived from subcutaneous stromal cells, the increase in Erk-1 and Erk-2 phosphorylation in response to insulin reached a tenfold peak after $15 \mathrm{~min}$ ( $p=0.001$ vs basal for both Erk-1 and Erk-2), gradually decreasing toward basal levels after $120 \mathrm{~min}$ (Fig. 6a,b). By contrast, in omental adipocytes, insulin stimulation induced a more rapid and transient increase of Erk phosphorylation, which peaked at $5 \mathrm{~min}(p=0.001 \mathrm{vs}$ basal for both Erk-1 and Erk-2) and then returned to basal levels at $120 \mathrm{~min}$ (Fig. 6a,b). Consequently, in omental compared with subcutaneous 
a

SC

0
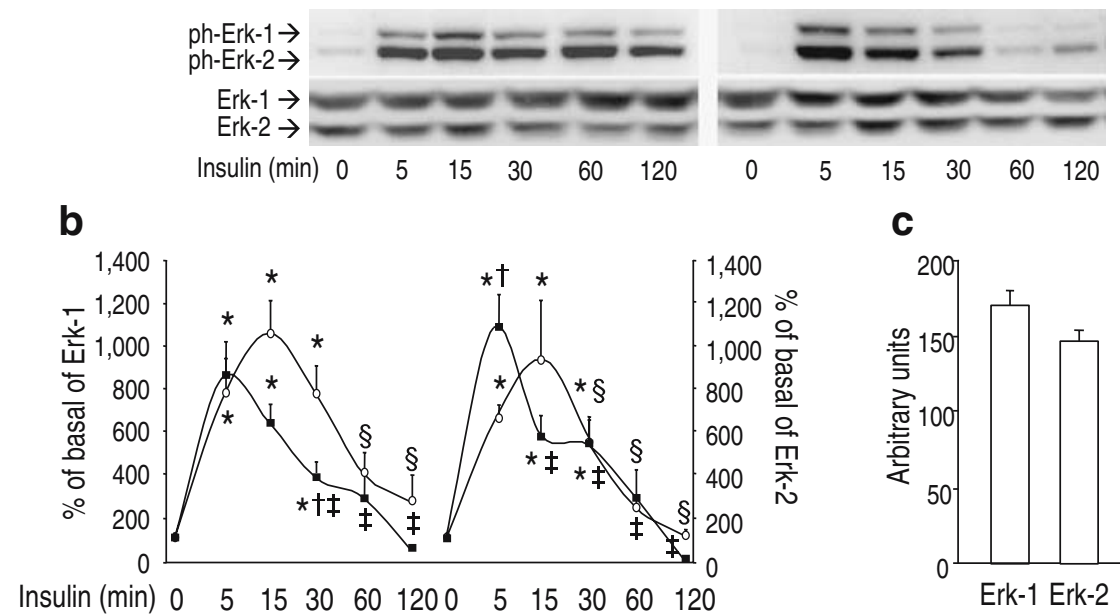

C

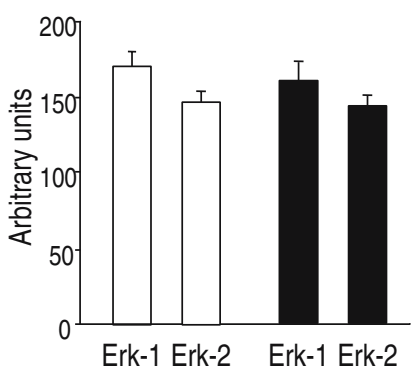

Fig. 6 Activation of Erk in subcutaneous and omental adipocytes differentiated in vitro from precursor stromal cells. Subcutaneous (SC, white bars/circles) and omental (O, black bars/squares) adipocytes were studied in the absence or presence of $10 \mathrm{nmol} / 1$ insulin for the indicated times. Cell lysates were subjected to immunoblotting with antibodies specific to total or phosphorylated forms of Erk-1 and Erk2. a Representative immunoblots of Erk- $1 / 2$ phosphorylation (ph) and protein content. b Quantitation of the phosphorylation levels of Erk

isoforms during insulin stimulation. c Quantitation of total Erk-1 and Erk-2 protein content; values of basal and insulin-stimulated samples pooled together. Data are expressed as mean \pm SE of paired subcutaneous and omental adipocytes from eight independent participants. ${ }^{*} p<0.05$ vs basal; ${ }^{\dagger} p<0.05$ vs corresponding time-point in SC adipocytes; ${ }^{\$} p<0.05$ vs 15 min time-point in SC adipocytes; ${ }^{+} p<0.05$ vs 5 min time-point in omental adipocytes

adipocytes, Erk-2 phosphorylation was significantly higher at 5 min and Erk-1 phosphorylation was significantly lower at $30 \min (p=0.039$ and $p=0.014$ vs subcutaneous adipocytes at the named time-points, respectively) (Fig. 6b), depicting different Erk activation kinetics in the two fat cell populations. The earlier and more transient insulin-induced phosphorylation of Erk proteins in omental as compared with subcutaneous adipocytes was similar to the kinetics of IR, IRS and Akt phosphorylation (Figs. 4 and 5). Interestingly, total protein levels of Erk-1 or Erk-2 were similar in subcutaneous and omental adipocytes $(p=0.89)$ (Fig. 6a,c), so differences in Erk protein activation could not be accounted for by different cellular protein content of these mitogen-activated protein kinases.

\section{Discussion}

The biological diversity of adipose tissue depots has become a fundamental issue in recent years, in light of its potential impact on human health [2]. It is known that visceral adipose tissue is morphologically and functionally different from subcutaneous adipose tissue [8]. Depot-related variations have been described for a variety of biological endpoints, such as activation of the insulin signalling pathway [12], glucose uptake responses $[13,14]$ and adiponectin secretion [19]. However, the precise mechanisms underlying the observed differences remain unclear. It has been proposed that extrinsic factors, including depot-specific blood flow, cell density, cell heterogeneity and/or innervation [20] could contribute to distinct gene expression patterns and metabolic profiles in adipocytes of different anatomical regions. In this study, we demonstrate that depot-related differences in gene expression and adiponectin secretion are still evident when adipocyte precursor cells are isolated from bioptic adipose tissue fragments, differentiated in vitro and studied several weeks after removal from their original tissue environment. In addition, after insulin stimulation, adipocytes differentiated from omental stromal cells show earlier and more transient kinetics of activation of multiple signalling intermediates, including the IR, IRS proteins, Akt and Erk-1/2, as well as significantly greater glucose transport rates than adipocytes derived from subcutaneous stromal precursors. Interestingly, the results on insulin signalling and glucose uptake responses in this in vitro experimental system resemble previous observations in whole fat tissues in vivo $[12,13]$. These findings suggest that the biological differences of the two fat depots depend, at least in part, on innate characteristics of subcutaneous and omental adipose cells. This concept is supported by the recent demonstration that preadipocytes from subcutaneous and omental fat tissues retain fat depot-specific dynamic characteristics and gene expression patterns even after 40 cell doublings [16].

Adipogenesis involves the sequential induction of multiple transcription factors, acquisition of specific metabolic com- 
petences and ability to synthesise and secrete fat-specific proteins. In our experimental system, both subcutaneous and visceral adipocytes exhibited accumulation of cytoplasmic triacylglycerol droplets and markedly increased expression of $P P A R \gamma, C / E B P \alpha, A P 2$, adiponectin and GLUT4 mRNAs, as compared with precursor stromal cells, in line with previous reports [16]. Altogether, these findings indicate that the subcutaneous and visceral cells completed the differentiation process into mature adipocytes.

$C / E B P \alpha, A P 2$ and PPAR $\gamma$ cross-regulate each other's expression and govern the entire adipogenic programme, by coordinating the expression of lipogenic enzymes and other adipocyte-specific genes [21, 22], including glycerophosphate dehydrogenase, adiponectin and GLUT4 [23]. In contrast to $C / E B P \beta$ (also known as $C E B P B$ ) and $C / E B P \delta$ (also known as $C E B P D$ ), whose mRNA levels increase transiently at the onset of adipocyte differentiation, $C / E B P \alpha$ is induced at a subsequent phase and remains expressed at high levels in mature adipocytes [23]. Adiponectin mRNA levels rise immediately after the increase of $C / E B P \alpha$, remaining at high levels in mature adipocytes. In this study, mRNA levels of $C / E B P \alpha$ and $A P 2$ were expressed at higher levels in subcutaneous adipocytes than in omental adipose cells, in line with a previous report [16]; adiponectin mRNA levels were also higher in subcutaneous adipocytes and in subcutaneous fat biopsies (Fig. 2). This result is in contrast with the results of others [24], who found no differences in adiponectin gene expression in freshly isolated subcutaneous and omental human adipocytes. However, it should be noted that subjects in that study had a mean BMI of $46.4 \pm 2.0 \mathrm{~kg} / \mathrm{m}^{2}$ (range, $32-58.4 \mathrm{~kg} / \mathrm{m}^{2}$ ). Since CCAAT/enhancer binding protein $(\mathrm{C} / \mathrm{EBP})$, alpha $(\mathrm{C} / \mathrm{EBP} \alpha)$ is an important transcription factor for the human adiponectin gene [25], the differences in adiponectin gene expression in subcutaneous compared with omental adipocytes may be related to the different levels of $C / E B P \alpha$ mRNA.

Using RT-PCR primers that do not discriminate between the two isoforms of peroxisome proliferator-activated receptor gamma (PPAR $\gamma$ ), i.e. $\gamma 1$ and $\gamma 2, P P A R \gamma$ mRNA levels were found to be similar in subcutaneous and omental adipocytes, in line with results obtained in both whole fat tissue specimens and isolated adipocytes in this study and elsewhere [26-28]. This finding is apparently in contrast with the reportedly higher levels of PPAR $\gamma$ found in subcutaneous compared with visceral adipocytes differentiated in vitro in the study by Tchkonia et al. [16]. However, in that previous study adipocytes were analysed 15 days after induction of differentiation, whereas in this study they were studied after a longer time.

Similarly to PPAR $\gamma$, mRNA expression levels of GLUT1 and GLUT4 did not vary significantly between omental and subcutaneous adipocytes. However, GLUT4 mRNA was found to be slightly higher in subcutaneous than in omental adipocytes, even though this difference was not statistically significant, a finding which is analogous to previous observations in adipose tissue fragments isolated from subcutaneous and omental fat depots [29]. On the other hand, GLUT1 was already expressed at high levels in preadipocytes and did not show prominent changes with differentiation, in line with similar observations in 3T3-L1 cells [30].

In previous studies on isolated fat cells, visceral adipocytes exhibited a higher adiponectin release than subcutaneous adipocytes, both in the basal state (although not significantly) and following insulin or thiazolidinedione incubation [19]. In this study, the rates of adiponectin secretion were approximately threefold higher in the adipocytes differentiated from omental than from subcutaneous precursors. The apparent discrepancy between changes in adiponectin mRNA expression, which was found to be higher in subcutaneous-derived adipocytes and in subcutaneous fat biopsies (Fig. 2), and changes in adiponectin protein release in the culture medium, which was higher in omental cells (Fig. 3a), suggests that adiponectin gene expression and protein secretion may be regulated differently in specific fat depots. Indeed, adiponectin gene expression is under the transcriptional control of $\mathrm{C} / \mathrm{EBP} \alpha$ [25], which was found to be expressed at higher levels in subcutaneous than in omental adipocytes (Fig. 2a), whereas adiponectin secretion can be regulated by other factors, including insulin, as shown in 3T3-L1 adipocytes $[31,32]$. Whether more rapid insulin signalling responses, as observed in omental compared with subcutaneous adipocytes, may result in faster adiponectin secretion rates will be the focus of future studies. Additionally, it has been recently demonstrated that the intracellular deacetylase sirtuin (silent mating type information regulation 2 homologue) 1 (S. cerevisiae) (SIRT1) stimulates adiponectin gene transcription by cooperating with forkhead box $\mathrm{O} 1$ (FOXO1) and $\mathrm{C} / \mathrm{EBP} \alpha$ [33], while it suppresses adiponectin secretion [34]. Thus, it will also be interesting to investigate whether SIRT1 may be differently expressed and/or activated in visceral than in subcutaneous fat depots.

Insulin-stimulated glucose transport was also found to be higher, by approximately two- to threefold, in omental than in subcutaneous adipocytes. This finding is again in accordance with earlier studies in whole fat tissues in humans in vivo [13] and isolated human adipocytes in vitro [14], which demonstrated twofold greater rates of insulinstimulated glucose uptake in the visceral fat depot. Thus, regional variations between visceral and subcutaneous adipocytes, including differences in adiponectin release and glucose metabolism, appear to be preserved in cultures of stromal cells differentiated into mature adipocytes in vitro, suggesting the existence of intrinsic traits of subcutaneous- and visceral-oriented fat cells. 
In omental adipocytes, insulin induced a more rapid and transient activation of IR, IRS proteins, Akt and Erk-1/2 compared with subcutaneous cells, in which activation of these proteins was more gradual and somewhat sustained. These results strikingly recall the distinct temporal profile of activation of the same insulin signalling proteins observed in human omental and subcutaneous fat depots in response to insulin administration intravenously in vivo [12]. In that previous study, omental fat showed maximum increases in IR, Akt and Erk phosphorylation 6 min after insulin injection, with subsequent decreases at $30 \mathrm{~min}$, whereas in subcutaneous fat activation of insulin signalling reached its maximum $30 \mathrm{~min}$ after insulin stimulation [12]. The phosphorylation levels of specific signalling proteins, such as the IR and Erk-1/2, were found to be higher in omental than in subcutaneous fat in the in vivo study [12], whereas in this study the omental and subcutaneous adipocytes differentiated in vitro exhibited different temporal profiles but similar magnitude of activation of all signalling reactions. It should be noted, however, that omental fat tissue as a whole is characterised by higher protein content of IR and Erk-1/2 than that found in subcutaneous fat and that the differences in the extent of phosphorylation between the two fat depots are minimised when they are normalised per amount of protein [12]. The more rapid and less persistent phosphorylation profile of the IR and downstream signalling molecules in response to insulin stimulation in omental adipocytes could be potentially explained by higher levels of specific phosphotyrosine phosphatases. However, we found comparable protein content of PTP-1B, SHIP-2 or PTEN in subcutaneous vs omental adipocytes (see ESM Fig. 1). It is possible that differences in specific phosphatase activities and/or the involvement of other, as yet unknown phosphatases, may contribute to the different signalling kinetics in the two fat cell types.

In conclusion, even when differentiated in vitro, subcutaneous and omental adipose cells retain fat depot-specific characteristics in gene expression, adiponectin release, and insulin signalling and action. It is tempting to speculate that there may be an early commitment of fat precursor stromal cells towards a depot-oriented differentiation, which may precede and be independent of extrinsic influences from the tissue environment. Additional studies are needed to elucidate how extrinsic and environmental factors interact with the innate properties of adipocytes under normal conditions and in disease states.

Acknowledgements This work was supported by grants from the Ministero dell'Università e Ricerca (Italy), the Cofinlab 2000 - Centro di Eccellenza 'Genomica comparata: geni coinvolti in processi fisiopatologici in campo biomedico e agrario' (Italy) and grants from Pfizer Italia (ARADO Programme) and Novo Nordisk (LIBRA Programme) to F. Giorgino.
Duality of interest The authors declare that there is no duality of interest associated with this manuscript.

\section{References}

1. Evans DJ, Hoffmann RG, Kalkhoff RK, Kissebah AH (2001) Relationship of body fat topography to insulin sensitivity and metabolic profiles in premenopausal women. Metabolism 33:68-75

2. Despres JP (2001) Health consequences of visceral obesity. Ann Med 33:534-541

3. Gastaldelli A, Miyazaki Y, Pettiti M et al (2001) Metabolic effects of visceral fat accumulation in type 2 diabetes. J Clin Endocrinol Metab 87:5098-5103

4. Gabriely I, Ma XH, Yang XM et al (2002) Removal of visceral fat prevents insulin resistance and glucose intolerance of aging: an adipokine-mediated process? Diabetes 51:2951-2958

5. Thorne A, Lonnqvist F, Apelman J, Hellers G, Arner P (2002) A pilot study of long-term effects of a novel obesity treatment: omentectomy in connection with adjustable gastric banding. Int $\mathbf{J}$ Obes Relat Metab Disord 26:193-199

6. Cases JA, Barzilai N (2000) The regulation of body fat distribution and the modulation of insulin action. Int $\mathrm{J}$ Obes Relat Metab Disord 24:S63-S66

7. Klein S, Fontana L, Young VL et al (2004) Absence of an effect of liposuction on insulin action and risk factors for coronary heart disease. N Engl J Med 350:2549-2557

8. Wajchenberg BL (2000) Subcutaneous and visceral adipose tissue: their relation to the metabolic syndrome. Endocr Rev 21:697-738

9. Giorgino F, Laviola L, Eriksson JW (2005) Regional differences of insulin action in adipose tissue: insights from in vivo and in vitro studies. Acta Physiol Scand 183:13-30

10. Zierath JR, Livingston JN, Thorne A et al (1998) Regional difference in insulin inhibition of non-esterified fatty acid release from human adipocytes: relation to insulin receptor phosphorylation and intracellular signalling through the insulin receptor substrate-1 pathway. Diabetologia 41:1343-1354

11. Sjoholm K, Palming J, Olofsson LE et al (2005) A microarray search for genes predominantly expressed in human omental adipocytes: adipose tissue as a major production site of serum amyloid A. J Clin Endocrinol Metab 90:2233-2239

12. Laviola L, Perrini S, Cignarelli A et al (2006) Insulin signaling in human visceral and subcutaneous adipose tissue in vivo. Diabetes 55:952-961

13. Virtanen KA, Lonnroth P, Parkkola R et al (2002) Glucose uptake and perfusion in subcutaneous and visceral adipose tissue during insulin stimulation in nonobese and obese humans. J Clin Endocrinol Metab 87:3902-3910

14. Lundgren M, Buren J, Ruge T, Myrnas T, Eriksson JW (2004) Glucocorticoids down-regulate glucose uptake capacity and insulin-signaling proteins in omental but not subcutaneous human adipocytes. J Clin Endocrinol Metab 89:2989-2997

15. Rodbell M (1964) Metabolism of isolated fat cells. Effects of hormones on glucose metabolism and lipolysis. J Biol Chem 239:375-380

16. Tchkonia T, Giorgadze N, Pirtskhalava T et al (2006) Fat depotspecific characteristics are retained in strains derived from single human preadipocytes. Diabetes 55:2571-2578

17. Kramer DK, Al-Khalili L, Perrini S et al (2005) Direct activation of glucose transport in primary human myotubes after activation of peroxisome proliferator-activated receptor delta. Diabetes 54:1157-1163

18. Perrini S, Natalicchio A, Laviola L et al (2004) Dehydroepiandrosterone stimulates glucose uptake in human and murine adipocytes by inducing GLUT1 and GLUT4 translocation to the plasma membrane. Diabetes 53:41-52 
19. Motoshima H, Wu X, Sinha MK et al (2000) Differential regulation of adiponectin secretion from cultured human omental and subcutaneous adipocytes: effects of insulin and rosiglitazone. J Clin Endocrinol Metab 87:5662-5667

20. Hausman GJ, Hausman DB (2006) Search for the preadipocyte progenitor cell. J Clin Invest 116:3103-3106

21. Rosen ED, Walkey CJ, Puigserver P, Spiegelman BM (2000) Transcriptional regulation of adipogenesis. Genes Dev 14:1293-1307

22. Gregoire FM, Smas CM, Sul HS (1998) Understanding adipocyte differentiation. Physiol Rev 78:783-809

23. Tang QQ, Zhang JW, Lane DM (2004) Sequential gene promoter interactions by $\mathrm{C} / \mathrm{EBPbeta}, \mathrm{C} / \mathrm{EBPalpha}$, and PPARgamma during adipogenesis. Biochem Biophys Res Commun 318:213-218

24. Degawa-Yamauchi M, Moss KA, Bovenkerk JE et al (2005) Regulation of adiponectin expression in human adipocytes: effects of adiposity, glucocorticoids, and tumor necrosis factor alpha. Obes Res 13:662-669

25. Qiao L, Maclean PS, Schaack J et al (2005) C/EBPalpha regulates human adiponectin gene transcription through an intronic enhancer. Diabetes 54:1744-1754

26. Quinkler M, Bujalska IJ, Tomlinson JW, Smith DM, Stewart PM (2006) Depot-specific prostaglandin synthesis in human adipose tissue: a novel possible mechanism of adipogenesis. Gene 380:137-143

27. Bower JF, Davis JM, Hao E, Barakat HA (2006) Differences in transport of fatty acids and expression of fatty acid transporting proteins in adipose tissue of obese black and white women. Am J Physiol Endocrinol Metab 290:E87-E91

28. Montague CT, Prins JB, Sanders L et al (1998) Depot-related gene expression in human subcutaneous and omental adipocytes. Diabetes 47:1384-1391

29. Marette A, Mauriege P, Marcotte B et al (1997) Regional variation in adipose tissue insulin action and GLUT4 glucose transporter expression in severely obese premenopausal women. Diabetologia 40:590-598

30. Weiland M, Schermann A, Schmidt WE et al (1990) Development of the hormone-sensitive glucose transport activity in differentiating $3 \mathrm{~T} 3 \mathrm{~L} 1$ murine fibroblasts. Role of the two transporter species and their subcellular localization. Biochem J 270:331-336

31. Scherer PE, Williams S, Fogliano M et al (1995) A novel serum protein similar to $\mathrm{C} 1 \mathrm{q}$, produced exclusively in adipocytes. J Biol Chem 270:26746-26749

32. Bogan JS, Lodish HF (1999) Two compartments for insulinstimulated exocytosis in 3T3-L1 adipocytes defined by endogenous ACRP30 and GLUT4. J Cell Biol 146:609-620

33. Qiao L, Shao J (2006) SIRT1 regulates adiponectin gene expression through Foxo1-C/enhancer-binding protein alpha transcriptional complex. J Biol Chem 281:39915-39924

34. Qiang L, Wang H, Farmer SR (2007) Adiponectin secretion is regulated by SIRT1 and the endoplasmic reticulum oxidoreductase Ero1-L alpha. Mol Cell Biol 27:4698-4707 